\title{
Ermittlung charakteristischer Spinnengemeinschaften von Biotoptypen am Beispiel der nordwestdeutschen Küstenregion
}

\author{
Oliver-D. FINCH \& Walter SCHULTZ
}

\begin{abstract}
Investigation of characteristic spider communities of biotope types taking the northwest German coastal area as an example. A classification method for ascertaining characteristical spider communities of different biotope types is presented. Based on a current list of biotope types all data available from a certain area about the spatial distribution of spiders were fed into a data bank and analysed. A largely standardized method has been developed which allows to classify the spider community of each biotope type. The classification is based on a presence/absence matrix and a comparison between the grades of presence in different biotope types.
\end{abstract}

Key words: spider communities, biotope types, classification method

\section{EINLEITUNG}

Um zu einer verbesserten Nutzbarkeit von ökofaunistischen Daten in der Planung zu gelangen, wird allgemein die Erstellung von regionalisierten und lebensraumbezogenen Standard-, Charakter- bzw. Leitartenlisten gefordert (vgl.u.a. RECK 1990, REUSCH 1995, RIECKEN 1992, RIECKEN et al. 1995). Solche Listen können einen Bezugsrahmen für leitbildorientierte naturschutzfachliche Bewertungen darstellen und bei der Entwicklung naturraumspezifischer Leitbilder helfen, da sie generalisierte (potentielle) Ausprägungsspektren der Lebensgemeinschaft des zu untersuchenden Objektes aufzeigen (vgl. PLACHTER 1994). Wenn bei der Erstellung regionalisierter Leitartenlisten "Biotoptypen" (vgl. z.B. DRACHENFELS 1992, RIECKEN etal. 1994) als Raumeinheiten zugrunde gelegt werden, so ist damit eine maßgebliche, planungsrelevante, räumliche Bezugsgröße gewählt, denn Biotoptypen werden für landschaftsökologische Gutachten regelmäßig kartiert. Da biotoptypenbezogene Charakterartenlisten als Vergleichsbasis für Artenbestände konkreter Untersuchungen heranziehbar sind und bisherige, z.T. unbefriedigende Bewertungsparameter wie Rote Listen, Artenzahlen etc. sinnvoll ergänzen, können sie in der Planungspraxis 
zu einer wesentlichen Verbesserung naturschutzfachlicher Bewertungen beitragen. Das hier vorgestellte Verfahren zur Aufarbeitung arachnologischer Daten zu regionalisierten, lebensraumbezogenen Leitartenlisten der Spinnen dernordwestdeutschen Küstenregion (vgl. SCHULTZ \& FINCH 1996) stellt eine Möglichkeit dar, charakteristische Artenkomplexe näher zu typisieren (vgl. u.a. BRAUKMANN 1987, FLADE 1995, HILDEBRANDT \& HANDKE 1996, RIECKEN \& BLAB 1989).

\section{GRUNDSATZÜBERLEGUNGEN}

Oberster Grundsatz für die Erstellung von Standard-Artenlisten ist die Regionalisierung, d.h. die Begrenzung auf einen bestimmten Raum. Durch ausschließliche Verwendung regionaler Ausgangsdaten soll z.B. das Prinzip der regionalen Stenotopie (vgl. TISCHLER 1993) berücksichtigt werden. Für den zu bearbeitenden Raum sollte eine relativ breite Datenbasis vorhanden sein. Zudem soll mit der hier präsentierten Methode ein möglichst geringer Einfluß des Bearbeiters bezüglich der Artenklassifizierungen erreicht werden. Von wesentlicher Bedeutung ist des weiteren die Transparenz der Auswertungsschritte, die Reproduzierbarkeit der Ergebnisse und die Fortschreibbarkeit - also die Integrationsmöglichkeit zukünftiger Untersuchungen.

\section{DATENERMITTLUNG}

Das hier vorgestellte Auswertungsverfahren zu Erstellung arachnologischer Leitartenlisten wurde bisher für die nordwestdeutsche Küstenregion angewendet (SCHULTZ \& FINCH 1996). Für diesen Raum kann die Datengrundlage als vergleichweise gut bezeichnet werden (vgl. FRÜND et al. 1994). Das Gebiet umfaßt die deutsche Nordseeküste von Borkum bis Sylt und die schleswig-holsteinische Ostseeküste. Alle Landschaftsräume in unmittelbarer Nähe zum offenen Meer (z.B. auch Polder, Köge)werden als küstenzugehörig aufgefaßt. Die systematische Auswertung der arachnofaunistischen Literatur basiert auf dem Literaturverzeichnis zur "Checkliste" von FRÜND et al. (1994). Unberücksichtigt blieb sogenannte "Graue Literatur" mit Ausnahme eigener Gutachten. Es konnten 31 Quellen erschlossen werden; durch Teilung dieser Quellen bei disjunkten Untersuchungsräumen erhöhte sich die Quellenanzahl auf 55. Insgesamt fanden 301 Spinnenarten Berücksichtigung. Die Region wurde nach der aktuellen 
Roten Liste gefährdeter Biotoptypen der Bundesrepublik (RIECKEN et al. 1994) in Lebensraumtypen gegliedert. Abänderungen bzw. Ergänzungen der Definitionen waren für einige Typen - v.a. Biotopkomplexe - erforderlich. Das hierarchische System der Biotoptypengliederung von RIECKEN et al. (1994) wurde beibehalten, wodurch eine Unterscheidung von Biotoptypen in Obereinheiten und Untereinheiten möglich wird (vgl. Code-Nummern in Tab. 1). Hierbei stellen Obereinheiten zum überwiegenden Teil Biotopkomplexe dar. Für insgesamt 40 Biotoptypen wurden Standard-Artenlisten der Spinnenfauna erstellt. Abundanzangaben zu den einzelnen Arten mußten weitgehend unberücksichtigt bleiben, da den herangezogenen Untersuchungen unterschiedliche Erfassungsmethoden (i.w.S.) zu'grunde liegen, die keine Vergleiche der jeweiligen Individuendichten erlauben. Allerdings werden zur Absicherung der Ergebnisse auf oberen Biotoptypen-Ebenen Abundanzangaben ausgewählter Referenzuntersuchungen angeführt (für Salzwiesen z.B. BRÖRING et al. 1993) und bei der Artenklassifizierung berücksichtigt (vgl. Tab. 1).

\section{AUFBAU DER DATENBANK}

Anhand der Literaturangaben erfolgte eine Eintragung der Nachweise jeder Art pro Biotoptyp (vgl. Präsenz-Absenz-Matrix; Tab. 1). Zudem wurde eine Aufschlüsselung der Einträge bezüglich der jeweiligen Literaturquellen vorgenommen. Durch diese Verbindung zwischen Quellen und PräsenzEinträgen soll eine Fortschreibbarkeit der Artenlisten ermöglicht werden (vgl. Anhang in SCHULTZ\& FINCH 1996). Nichtalle Literaturangaben ließen sich bis zu unteren Biotoptypen-Ebenen aufschlüsseln, z.T. waren die Quellenangaben nur höheren hierarchischen Ebenen zuzuordnen. Insgesamt liegen den Auswertungen 9318 Dateneinträge zugrunde.

Durch das hierarchische System der Biotoptypeneinteilung in Ober-und Untereinheiten müssen Daten der Untereinheiten zum Teil auf nächsthöherer Ebene zusammengefaßt werden, da es sich um einen gemeinsamen Obertypus handelt, der die Untereinheiten einschließt. Eine einfache summarische Betrachtung der Stetigkeitswerte einer Art von den Untereinheiten zu den Obereinheiten ist deshalb nicht möglich. Zur Verdeutlichung ein Beispiel: Von zwei Salzwiesen eines Untersuchungsraumes wird die eine beweidet, die andere ist ungenutzt. Auf der Ebene der Untereinheiten sind beide Wiesen getrennt als naturnahe bzw. als anthropozoogen überformte Salzwiese aufzufassen. Sie gehören aber beide zur Obereinheit der Salzwiesen und werden hier nur einmal berücksichtigt, da sie räumlich nicht deutlich separiert sind (z.B. durch ihre Lage auf einer Insel). 
Tab. 1: (Ausschnitt aus Tab. 2 in SCHULTZ \& FINCH (1996) als Beispiel) Die Spinnenfauna der nordwestdeutschen Küstenregion und ihre biotoptypenbezogene Verteilung (Biotoptypen, inkl. Biotoptyp-Code-Nummern und regionaler Gefährdung der einzelnen Typen - z.T. verändert - nach RIECKEN et al. (1994); Zahlenwerte = Anzahl der Nachweise je Spinnenart und Lebensraum)

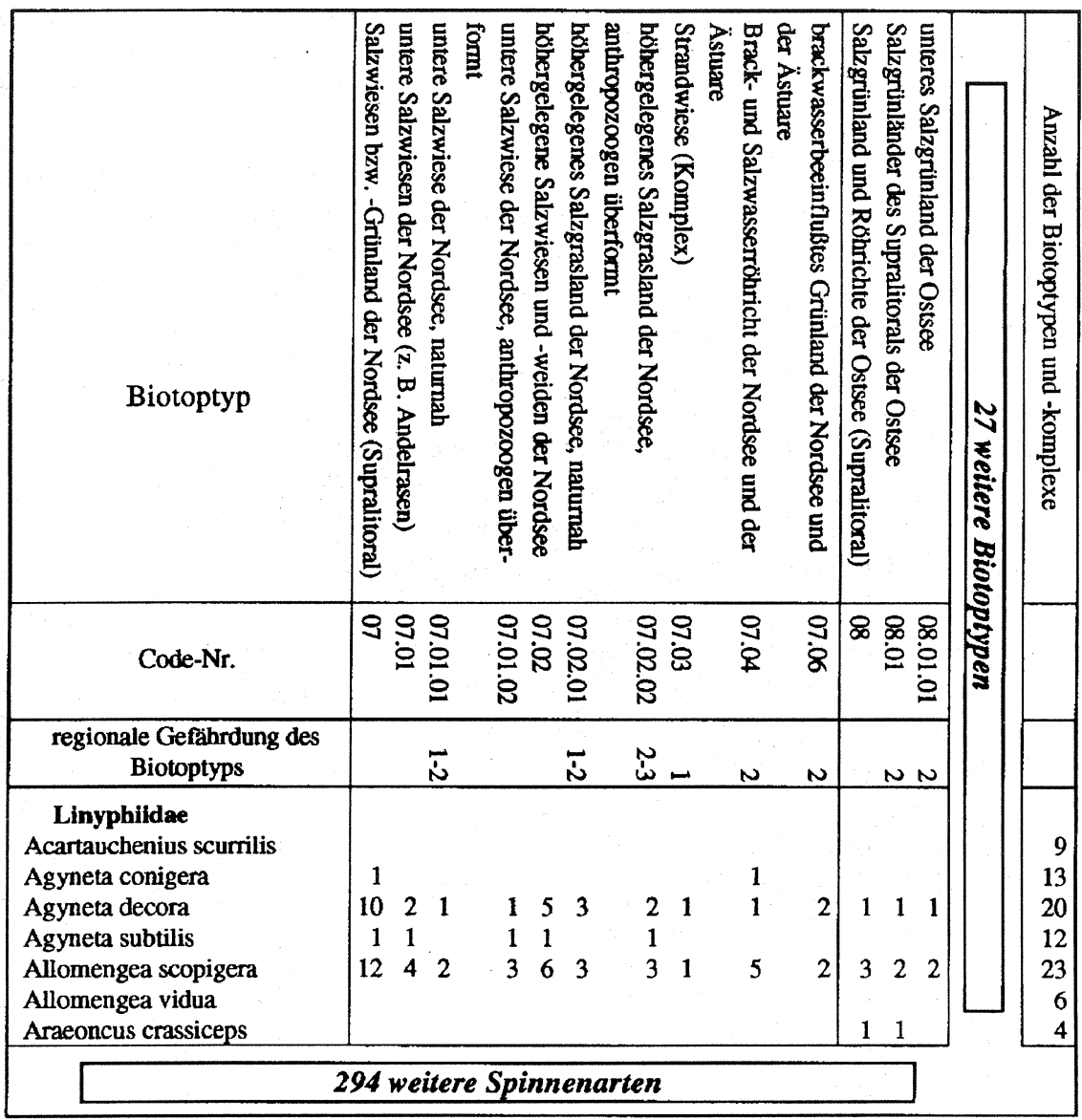


Durch die je Biotoptyp und Art vorgenommenen Präsenzwert-Einträge liegen Vergleichswerte für den Abgleich zwischen den verschiedenen Biotoptypen vor. Die Auswertung erfolgt anhand eines weitgehend formalisierten Verfahrens, das nachvollziehbare Klassifizierungen der Arten liefert.

Das Klassifizierungsverfahren basiert überwiegend auf einem Abgleich zwischen Präsenz-Graden. Der Präsenz-Grad (P.-G.) einer Art in einem Lebensraum wird ermittelt, indem der absolute Präsenz-Wert (P.) in Relation zur jeweiligen Quellenanzahl für den Biotoptyp gesetzt wird (P.-G. = P. x 100/ Quellenanzahl).

Um zu einer Gewichtung des Präsenz-Grades einer Spinnenart in einem Biotoptyp zu gelangen, wird ein operationalisierter Abgleich mit den PräsenzGraden der betreffenden Art in weiteren Biotoptypen (Fremd-BiotoptypPräsenz-Grad: F.-P.-G.) durchgeführt (s.u.). Die Berechnungen führen zu einer Werteskala bezüglich der Stetigkeit der Arten im betrachteten Biotoptyp im Vergleich zu weiteren Lebensraumtypen. Die Auswahl der zum Abgleich jeweils herangezogenen "Fremd-Biotoptypen" richtet sich nach der hierarchischen Ebene des betrachteten bzw. abzugleichenden Biotoptyps. Es wird somit zwischen Ober- und Untereinheiten (vgl. Biotoptyp-Codenummern) differenziert. Ein Abgleich wird ausschließlich zwischen KüstenBiotoptypen gleicher hierarchischer Ebene und nur zwischen deutlich unterscheidbaren Küstenlebensräumen durchgeführt. Beispielsweise werden Salzwiesen der Nordsee (Obereinheit, Code-Nr. 07) nicht gegen ihre Untereinheiten (z.B. untere Salzwiesen der Nordsee, Code-Nr. 07.01) oder auch nicht gegen die Ostsee-Salzwiesen (Code-Nr. 08) abgeglichen.

Zur Artenklassifizierung wurden zweiverschiedene Berechnungsschritte durchgeführt:

a. [Einzelvergleich] Für alle im jeweils betrachteten Biotoptyp präsenten Arten werden die einzelnen Präsenz-Grad-Differenzen(P.-G.-D.) zu jedem zum Abgleich herangezogenen "Fremd-Biotoptyp" gesondert berechnet (je Art:P.-G.-D. = P.-G. minus F.-P.-G.). Fürjede Art wirdalso derP.-G. im betrachteten Biotoptyp mit den P.-G.'s in weiteren ausgewählten Biotoptypen verglichen.

b. [summarischer Vergleich] Es wird je Art die Differenz zwischen dem Präsenz-Grad im betrachteten Biotoptyp und allen berücksichtigten und summierten "Fremd-Biotoptypen-Präsenz-Graden" der Küstenregion gebildet (je Art:P.-G.-D. = P.-G. minus $\Sigma$ F.-P.-G.).

Die Arten werden absteigend nach den jeweiligen Gesamt-Differenzen (P.-G.-D. nachb.) sortiert. 
Die Arten jedes Biotoptyps werden anhand ihres jeweiligen Spezialisierungsgrades in drei unterschiedliche Klassen eingeteilt, die wie folgt definiert werden:

- Leitarten (syn.: Charakterarten)als hoch charakteristische Arten, die im jeweils betrachteten Biotoptyp einen höheren Präsenz-Grad aufweisen als in allen weiteren zum Abgleich herangezogenen Biotopen zusammen (Ergebnis nach b. >0). Es handelt sich um Arten mit einer engen Lebensraumbindung, die besonders geeignet als Zeigerarten innerhalb der Gemeinschaften sind (vgl. FLADE 1995);

- sehr typische Arten, die im Einzelvergleich (!) der Biotoptypen im betrachteten Typ die höchste Präsenz aufweisen (Ergebnisse nach a. >0);

- typische Arten zeigen hohe Präsenz-Grade und teilweise sehr hohe Individuendichten im jeweils betrachteten Biotoptyp, allerdings auch in anderen Biotopen (regelmäßig im Lebensraumtyp erfaßbare und häufig relativ eurytope Arten).

\section{Klassengrenzen auf oberster Biotoptypen-Ebene (vgl. Tab. 2):}

Sehr heterogene Biotopkomplexe (oder genauer: Landschaftsräume, wie z.B. Küstendünen) machen Abweichungen von den Klassifizierungsregeln erforderlich, da sie stark unterschiedliche Lebensräume und Biozönosen umfassen (z.B.: anmoorige Dünentäler, Trockenrasen, Gehölze). Für die Küstendünen war es daher erforderlich, die Klassifizierungen zwischen der Obereinheit (Code-Nr. 10) und den entsprechenden Untereinheiten anhand der jeweiligen P.-G.-D.'s abzustimmen.

(1) Arten mitpositiven Differenz-Werten (P.-G.-D. nach b.) weisen im Bezugs-Biotoptyp einen höheren Präsenz-Grad (P.-G.) auf als in allen weiteren berücksichtigten Lebensraumtypen zusammen und werden in der Regel als Leitarten (L) oder sehr typische (+ T) Arten klassifiziert.

(2) Wenn der Präsenz-Grad einer Art im betrachteten Biotoptyp unter $10 \%$ liegt, wenn die Art in Nordwestdeutschland kennzeichnend für küstenfremde Festland-Biotoptypen (z.B. Wälder) ist und (oder) wenn die Dominanzanteile in eventuell vorhandenen ReferenzUntersuchungen unter $1 \%$ liegen, wird die Art nicht klassifiziert bzw. nicht in die Liste der Leitarten (i.w.S.) aufgenommen. Bei den betreffenden Arten handelt es sich i.d.R. um Einzelfunde bzw. biotopfremde Zufallsbesiedler.

(3) Arten mit negativen Präsenz-Grad-Differenzen (P.-G.-D. nach b.) bis minus $10 \%$ werden berücksichtigt, wenn der Präsenz-Grad im betrachteten Biotoptyp über $10 \%$ liegt, wenn in einer geeigneten Referenz-Untersuchung Dominanzanteile über $1 \%$ erreicht werden und (oder) wenn die Arten nicht kennzeichnend für andere Biotoptypen sind. Diese Araneiden 
sind in den Fremd-Biotoptypen zwar insgesamt etwas höher präsent (P.-G.-D. nach b.: $<0$ bis - 10) als im betrachteten Biotoptyp, weisen hier im Einzelvergleich der Biotoptypen (P.-G.-D. nach a.) aber oft die höchste Präsenz auf. Bei diesen Arten handelt es sich um sehr typische $(+T)$ bis typische $(T)$ Spinnen des Lebensraumtyps.

(4) Arten mit Präsenz-Grad-Differenzen (P.-G.-D. nach b.) kleiner minus $10 \%$ werden nur berücksichtigt, wenn derPräsenz-Grad im betrachteten Biotoptyp über $20 \%$ liegtund (oder) wenn in einer Referenz-Untersuchung Dominanzanteile $\geq 1 \%$ erreicht werden. Diese Spinnen werden i. d. R. als typische (T) Arten des Lebensraumtyps eingestuft.

(5) Arten mit Präsenz-Werten < 3 und (oder) Arten, die einem Biotoptyp nicht eindeutig zuzuordnen sind, werden in der jeweiligen Leitarten-Tabelle mit einem "?" aufgeführt.

Tab. 2: Klassengrenzen auf oberster Biotoptypenebene (vgl. Text; P.-G. = Präsenz-Grad,P.-G.-D. = Präsenz-Grad-Differenz, DOM. = Dominanzanteile in ausgewählten Referenzuntersuchungen)

\begin{tabular}{|l|c|c|c|c|}
\hline & P.-G.-D. nach b.) & P.-G.-D.nach a.) & $\begin{array}{c}\text { Experten- } \\
\text { einschätzung }\end{array}$ & weitere Kriterien \\
\hline Leitart(L) & immer $>0$ & immer $>0$ & $\begin{array}{c}\text { im Einzelfall } \\
\text { Abstufung } z u+T\end{array}$ & $\begin{array}{c}>10 \% \text { P.-G. } \\
(>1 \% \text { DOM. })\end{array}$ \\
\hline $\begin{array}{c}\text { sehr typische Art } \\
(+\mathrm{T})\end{array}$ & $\begin{array}{c}\text { überwiegend } \\
<0 \text { bis } \geq-10\end{array}$ & i.d.R. $>0$ & $\begin{array}{c}\text { im Einzelfall } \\
\text { Abstufung zu }+T\end{array}$ & $\begin{array}{c}>10 \% \text { P.-G. } \\
(>1 \% \text { DOM. })\end{array}$ \\
\hline typische Art (T) & $<-10$ & $\begin{array}{c}\text { nur ausnahmsweise } \\
\text { Aufwertung zu }+T\end{array}$ & $\begin{array}{c}>20 \% \text { P.-G. } \\
(\geq 1 \% \text { DOM. })\end{array}$ \\
\hline
\end{tabular}

\section{Klassengrenzen auf unteren Biotoptypen-Ebenen:}

Nur Arten, die für die jeweilige Obereinheit klassifiziert sind, können auch für die zugehörigen Untereinheiten klassifiziert werden. Ein Erreichen höherer Kategorien als in der entsprechenden Obereinheit ist nicht möglich. Als Fremd-Biotoptypen werden nur Untereinheiten innerhalb der gleichen Obereinheit herangezogen, der auch der gerade betrachtete Biotoptyp zuzuordnen ist.

(1) Alle Arten mit positiven Differenz-Werten (nach b.) erhalten i.d.R. die jeweiligen Klassifizierungen der Obereinheit.

(2) Arten mitPräsenz-Grad-Differenzen von $0 \%$ bis minus $10 \%$ weisen im Einzelvergleich der Biotoptypen häufig den höchsten Präsenz-Grad im betrachteten Typ auf und werden entsprechend den Klassifizierungen der Obereinheit alssehr typisch (+ T) bis fraglich (?) eingestuft .

(3) Spinnen mit Präsenz-Werten < 3 werden mit einem "?" versehen, da eine Klassifizierung aufgrund der unzureichenden Datenlage unsicher ist.

(4) Araneiden mit Differenz-Werten kleiner minus $10 \%$ werden nur berücksichtigt, wenn der jeweilige Präsenz-Wert im betrachteten Biotoptyp über 2 liegt. Die verbleibenden Arten werden i.d.R. als typische (T) Spinnen des Lebensraumes eingruppiert. 
Die Spinnenfauna der Salzwiesen bzw. des Salzgrünlandes der Nordsee, Code Nr. 07 (vgl. Tab. 3): Von den 134 in den Salzwiesen und im Salzgrünland der Nordsee nachgewiesenen Spinnenarten weisen 11 Arten in diesem Biotoptyp einen höheren Präsenz-Grad (P.-G.-D.) auf als in sämtlichen übrigen "Nicht-Salzwiesen-Biotoptypen". Für 9 dieser 11 Spinnenarten wird eine Einstufung als Leitart $(L)$ vorgenommen. Da für 3 (Collinsia distincta, Porrhomma oblitum, Silometopus incurvatus) dieser 11 Arten nur geringe Präsenz-Grade zu verzeichnen sind, werden diese Spinnen als potentielle, aber derzeit noch fragliche (? bzw. ? L) Leitarten eingruppiert. 6 weitere Arten sind als sehr typische (+ T) Spinnen des Biotoptyps zu bezeichnen. Diese Araneiden sind in den "Nicht-SalzwiesenBiotopen" der Küste zwar insgesamt etwas mehr präsent $(-0,3$ bis $-9,97)$ als in den Salzwiesen und im Salzgrünland der Nordsee, weisen im Einzelvergleich der Biotoptypen jedoch die höchste Präsenz im Lebensraumtyp 07 auf. Micaria lenzi ist nicht sicher einzustufen. 32 Arten sind typische ( $T$ ) Spinnen des Lebensraums 07, da sie dort überwiegend sehrhohe PräsenzGrade und teilweise ebenfalls sehr hohe Individuendichten aufweisen. Diese Arten werden regelmäßig im Biotoptyp 07 erfaßt und können dort durchaus dominant sein.

Insgesamt umfaßt die Leitartenliste der Spinnen der Salzwiesen und des Salzgrünlandes der Nordsee 50 Arten, von denen 46 Arten näher klassifiziert wurden. 10 (=20\%) der 50 Arten werden in der Roten Liste der Spinnen des deutschen Wattenmeerbereichs als potentiell bis stark gefährdet geführt (vgl. Tab. 3: Spalte R. L.). Von den gefährdeten Araneiden sind 6 als Leitarten für den Biotoptyp (bzw. -komplex) 07 ausgewiesen. Derhohe Anteil gefährdeter Arten spiegelt die Gefährdungssituation der Salzgrünlandkomplexe der Nordsee (vgl. RIECKEN et al. 1994) deutlich wider.

\section{ANMERKUNGEN}

Insgesamt konnten den einzelnen Biotopkomplexen der nordwestdeutschen Küstenregion bisher folgende Artenzahlen zugeordnet werden $(n=301)$ :

- Biotopkomplex Küstendünen: 275 Arten;

- Biotopkomplex Salzwiesen und -grünland der Nordsee: 134 Arten;

- Biotopkomplex Salzgrünland und Röhrichte der Ostsee: 75 Arten;

- Biotopkomplex Sände und Strände: 22 Arten. 
Tab. 3: (Beispiel aus SCHULTZ \& FINCH 1996) Liste der Leitarten (L), sehr typischen (+ T) und typischen ( $T$ ) Spinnen der (07) Salzwiesen bzw. des Salzwiesengrünlandes der Nordsee (R. L.: Einstufung der Arten in der Roten Liste der Spinnen des deutschen Wattenmeerbereichs (REINKE \& SCHULTZ 1995); DOM.: Dominanzwerte (nur Werte $\geq 1 \%$ ) nach ReferenzUntersuchungen (I, II) an der Nordseeküste; P. = absoluter Präsenz-Wert im Biotoptyp, P.-G. = Präsenz-Grad (P. x 100/Quellenanzahl f. Biotoptyp), F.-P.-G. = Fremd-Biotoptyp-Präsenz-Grad bzw. Präsenz-Grad in "NichtSalzwiesen-Lebensräumen" (hier: Summe der Präsenz-Grade aus Biotoptypen 09, 10, 34, 35.02, 41 und 44), P.-G.-D. = Präsenz-Grad-Differenz zwischen dem betrachteten Biotoptyp (hier: 07) und den Fremd-BiotoptypPräsenz-Graden (P.-G. minus F.-P.-G.)

\begin{tabular}{|c|c|c|c|c|c|c|c|c|}
\hline $\begin{array}{c}\text { Biotoptyp } 07 \\
\text { Quellenanzahl }\end{array}$ & & R. L. & DOM. I & DOM. II & P. & $\begin{array}{r}\text { P.-G. } \\
25\end{array}$ & $\begin{array}{r}\text { F.-P.-G. } \\
84\end{array}$ & P.-G.-D. \\
\hline $\begin{array}{l}\text { Argenna patula } \\
\text { Baryphyma duffeyi } \\
\text { Walckenaeria kochi } \\
\text { Allomengea scopigera } \\
\text { Walckenaeria vigilax } \\
\text { Agyneta decora } \\
\text { Ozyptila westringi } \\
\text { Porrhomma oblitum } \\
\text { Silometopus incurvatus } \\
\text { Robertus arundineti } \\
\text { Collinsia distincta }\end{array}$ & $\begin{array}{ll} & L \\
L \\
L \\
L \\
L \\
L \\
L \\
? \\
? \\
? \\
L \\
? \\
?\end{array}$ & $\begin{array}{l}p \\
3 \\
p \\
p \\
2 \\
3 \\
3\end{array}$ & m 14.8 & $2 / 11$ & $\begin{array}{r}11 \\
8 \\
13 \\
12 \\
12 \\
10 \\
3 \\
2 \\
1 \\
6 \\
2\end{array}$ & $\begin{array}{r}44 \\
32 \\
52 \\
48 \\
48 \\
40 \\
12 \\
8 \\
4 \\
24 \\
8\end{array}$ & $\begin{array}{r}27,7 \\
29,0 \\
34,8 \\
30,2 \\
5,9 \\
5,9 \\
2,9 \\
23,1 \\
7,1\end{array}$ & $\begin{array}{r}33,9 \\
32,0 \\
24,3 \\
19,0 \\
13,2 \\
9,8 \\
6,1 \\
2,1 \\
1,1 \\
0,9 \\
0,9\end{array}$ \\
\hline $\begin{array}{l}\text { Erigone longipalpis } \\
\text { Micaria lenzi } \\
\text { Enoplognatha mordax } \\
\text { Prinerigone vagans } \\
\text { Pocadicnemis juncea } \\
\text { Walckenaeria nudipalpis } \\
\text { Arctosa leopardus } \\
\text { Silometopus ambiguus } \\
\text { Porrhomma microphthal. }\end{array}$ & $\begin{array}{r}+T \\
? \\
+T \\
T \\
+T \\
T \\
+T \\
+T \\
+T \\
\end{array}$ & $\begin{array}{l}2 \\
3 \\
p\end{array}$ & $\begin{array}{r}45 / 38 \\
\\
-/ 3\end{array}$ & $\begin{array}{r}2 / 6 \\
-/ 4.8\end{array}$ & $\begin{array}{r}19 \\
1 \\
6 \\
4 \\
12 \\
5 \\
7 \\
9 \\
4\end{array}$ & $\begin{array}{r}76 \\
4 \\
24 \\
16 \\
48 \\
20 \\
28 \\
36 \\
16 \\
\end{array}$ & $\begin{array}{r}76,3 \\
5,9 \\
27,7 \\
21,4 \\
53,7 \\
26,1 \\
34,4 \\
44,5 \\
26,0 \\
\end{array}$ & $\begin{array}{l}-0,3 \\
-1,9 \\
-3,7 \\
-5,4 \\
-5,7 \\
-6,1 \\
-6,4 \\
-8,5 \\
-10,0\end{array}$ \\
\hline $\begin{array}{l}\text { Robertus lividus } \\
\text { Pardosa agrestis } \\
\text { Clubiona stagnatilis } \\
\text { Leptorhoptrum robustum } \\
\text { Silometopus reussi } \\
\text { Porrhomma pygmaeum }\end{array}$ & $\begin{array}{l}T \\
T \\
T \\
T \\
T \\
T\end{array}$ & & $\begin{array}{r}5 / 5 \\
\\
m 4 \\
m 1.2 \\
m 1.1 \\
\end{array}$ & $68 / 25$ & $\begin{array}{r}6 \\
21 \\
8 \\
9 \\
9 \\
4 \\
\end{array}$ & $\begin{array}{l}24 \\
84 \\
32 \\
36 \\
36 \\
16 \\
\end{array}$ & $\begin{array}{r}40,8 \\
104,0 \\
52,4 \\
64,6 \\
66,7 \\
47,9 \\
\end{array}$ & $\begin{array}{l}-16,8 \\
-20,0 \\
-20,4 \\
-28,6 \\
-30,7 \\
-31,9 \\
\end{array}$ \\
\hline \multicolumn{9}{|c|}{24 weitere (typische) Spinnenarten } \\
\hline
\end{tabular}


Die Artenklassifizierungen wurden innerhalb dieser Artenspektren durchgeführt (vgl. Abb. 1). Aufgrund der aktuellen Datengrundlage können die Auswertungen für die Küstenregion nur als erste Schritte zur Entwicklung raumbezogener Charakter-bzw. Zeigerarten-Systeme angesehen werden. Der auch für die Biotope der Küstenregion nach wie vor bestehende Forschungsbedarf wird z.B. über die in Teilbereichen ungenügende Datendichte deutlich. So konnten Artenklassifizierungen für die unteren Biotoptypeneinheiten aufgrund unzureichender Untersuchungsintensität nicht in jedem Falle erfolgen (vgl. SCHULTZ \& FINCH 1996). Als ein weiteres Problem bei der Erarbeitung des Leitartensystems für die nordwestdeutsche Küstenregion stellte sich die nicht gegebene Vergleichbarkeit der Abundanzangaben verschiedener Untersuchungen - denen unterschiedliche Erfassungsmethoden zugrunde liegen - heraus. Dies führt zu einem grundsätzlichen Informationsverlust hinsichtlich der räumlichen Verteilung der Spinnenfauna, der hier teilweise durch die Einbeziehung von Referenzuntersuchungen mit Abundanz-bzw. Dominanzangaben kompensiert wurde. Die vorwiegend auf Präsenzwerten beruhenden Artenklassifizierungen ließen die Definition von höchstens drei unterschiedlichen Kategorien (Klassen) sinnvoll erscheinen. Da es sich bei den Klassengrenzen um abgewägte Einteilungen handelt, sind diese natürlich diskutierbar. Um Fehleinschätzungen aufgrund starrer Klassengrenzen zu vermeiden, wurden die Klassifizierungsregeln (s.o. und Tab. 2) so aufgestellt, daß sie dem Bearbeiter im Einzelfall gestatten, korrigierend einzugreifen.

Um im Rahmen leitbildorientierter Bewertungen von Untersuchungsstandorten das Kriterium der "Repräsentanz, Vollständigkeit und Intaktheit" der untersuchten Biotope bzw. ihrer Zönosen beurteilen zu können, sind regionalisierte Leitartenlisten erforderlich (vgl. auch FLADE 1995). Über die in den Leitartenlisten verzeichneten Artenbestände der Biotope können Unterschiede zu konkreten Ist-Zuständen untersuchter Taxozönosen ersichtlich werden, die in Verbindung mit weiteren Standortfaktoren zu interpretieren sind (vgl. auch PLATEN 1995). Abweichungen von dem im Leitbild zu definierenden Soll-Inventar und das eventuelle Vorkommen von standortfremden Arten sind für die Indikation und die Ableitung von Naturschutzmaßnahmen zu nutzen.

Im Zusammenhang mit dem hier vorgestellten Verfahren zur Ermittlung von Leitartenlisten sei nachdrücklich vor einer Beschränkung zukünftiger Erfassungen auf die klassifizierten Arten gewarnt (vgl. auch PLATEN 1995, RECK 1990, SCHULTZ 1995). Es würde ein hohes Defizit an ökofaunistischer Information entstehen. Zudem wären differenzierte Standortbewertungen sowie ein Fortschreiben Roter Listen unmöglich. 


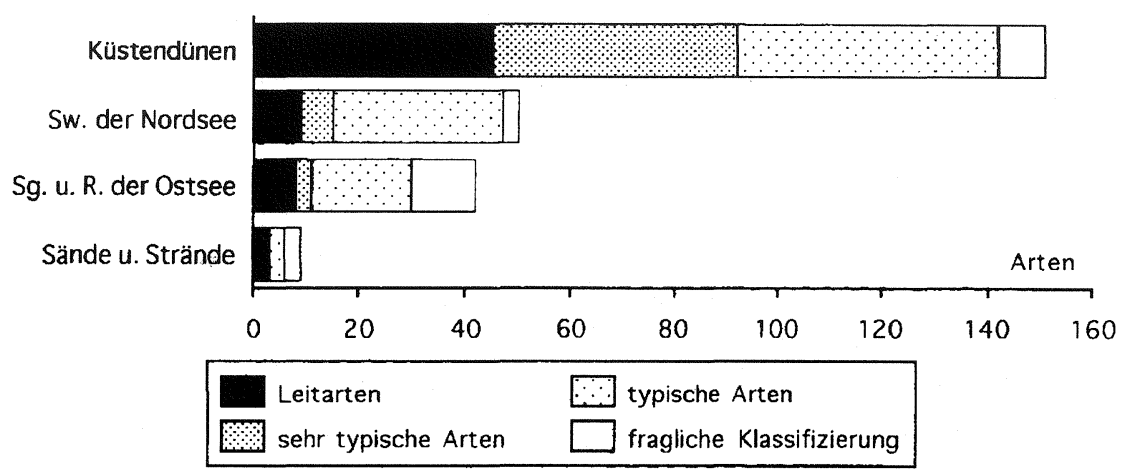

Abb. 1: Anzahl klassifizierter Arten in Biotopkomplexen der nordwestdeutschen Küstenregion (Sw. = Salzwiesen, Sg. u. R. = Salzgrünland und Röhrichte)

Danksagung: Wir danken Dr. H.-C.FRÜND (Osnabrück) sowie H.KRUMMEN und Dr. R.NIEDRINGHAUS (beide Oldenburg) für die kritische Durchsicht und die Anmerkungen zum Manuskript.

\section{LITERATUR}

BRAUCKMANN, U. (1987): Zoocoenologische und saprobiologische Beiträge zu einer allgemeinen Bachtypologie. - Arch. Hydrobiol., Ergebn. Limnol. 26: 1-355

BRÖRING, U., R.NIEDRINGHAUS \& W.SCHULTZ (1993): Ökofaunistische Zustandsanalyse und Bewertung von ehemaligen Kleientnahmestellen des Jadebusens und angrenzender Vergleichsflächen anhand der Spinnen-, Wanzen- und Zikadenfauna. - Gutachten i. A. des NLÖ, Forschungsstelle Küste, Norderney/Wilhelmshaven

DRACHENFELS, O. von (Bearb.) (1992): Kartierschlüssel für Biotoptypen in Niedersachsen unter besonderer Berücksichtigung der nach $\S 28$ a NNatG geschützeten Biotope. Stand Oktober 1992. - Naturschutz u. Landschaftspfl. Niedersachsen, Heft A/4: 1-168

FLADE, M. (1995): Aufbereitung und Bewertung vogelkundlicher Daten für die Landschaftsplanungunterbesonderer Berücksichtigung des Leitartenmodells.-Schr.-R. f. Landschaftspfl. u. Natursch. 43: 107-146

FRÜND, H.-C., J.GRABO, H.-D.REINKE, H.-B.SCHIKORA\& W.SCHULTZ (1994): Verzeichnis der Spinnen (Araneae) des nordwestdeutschen Tieflandes und Schleswig-Holsteins. - Arachnol. Mitt. 8: 1-46

HILDEBRANDT, J.\& K.HANDKE (1996): Biotoptypen des Grünlandes und deren Wirbellosenfauna-Versuch einer Zuordnung für den Bremer Raum. -Bremer Beitr. Naturkde. Natursch. 1: 83-94

PLACHTER, H. (1994): Methodische Rahmenbedingungen für synoptische Bewertungsverfahren im Naturschutz. - Z. Ökologie u. Naturschutz 3: 87-106 
PLATEN, R. (1995): Zeigerwerte für Laufkäfer und Spinnen - eine Alternative zu herkömmlichen Bewertungssystemen? - Schr.-R. f. Landschaftspfl. u. Natursch. 43: 317-328

RECK, H. (1990): Zur Auswahl von Tiergruppen als Biodeskriptoren für den tierökologischen Fachbeitrag zu Eingriffsplanungen. - Schr.-R. f. Landschaftspfl. u. Natursch. 32: 99-119 REINKE, H.-D. \& W.SCHULTZ (1995): Rote Liste der Spinnen (Araneae) des deutschen Wattenmeerbereichs. - Schr.-R. f. Landschaftspfl. u. Natursch. 44: 77-81

REUSCH, H. (1995): Planungsrelevante Aufbereitung und Bewertung faunistisch-ökologischer Daten vom Makrozoobenthon in Fließgewässern. - Schr.-R. f. Landschaftspfl. u. Natursch. 43: 31-43

RIECKEN, U. (1992): Planungsbezogene Bioindikation durch Tierarten und Tiergruppen. - Schr.-R. f. Landschaftspfl. u. Natursch. 36: 1-187

RIECKEN, U. \& J.BLAB (1989): Biotope der Tiere in Mitteleuropa. - Naturschutz aktuell 7: $1-123$

RIECKEN, U., U.RIES \& A.SSYMANK (1994): Rote Liste der gefährdeten Biotoptypen der Bundesrepublik Deutschland. - Schr.-R. f. Landschaftspfl. u. Natursch. 41: 1-184

RIECKEN, U., E.SCHRÖDER \& P.FINCK (1995): Mindestanforderungen an die planungsverwertbare Aufbereitung biologischer Daten im Rahmen naturschutzrelevanter Planungen. - Schr.-R. f. Landschaftspfl. u. Natursch. 43: 411-427

SCHULTZ, W. (1995): Zur Effektivität von Bodenfallen-Minimal-Erfassungsprogrammen. - Mitt. Dtsch. Ges. allg. angew. Ent. 10: 353-356

SCHULTZ, W. \& O.-D.FINCH (1996): Biotoptypenbezogene Verteilung der Spinnenfauna der nordwestdeutschen Küstenregion. - Cuvillier Verlag, Göttingen

TISCHLER, W. (1993): Einführung in die Ökologie. - Fischer Verlag, Stuttgart

Oliver-D. FINCH \& Walter SCHULTZ, Universität Oldenburg, FB 7 / AG Terr. Ökol., Postfach 2503, D - 26111 Oldenburg 